to 24 years. This study aims to evaluate the knowledge regarding PEP amongst young university students.

Methods Cross section study carried out from August to September 2016 among Health, Education and Exact Sciences students at the State University of Bahia. Socio-epidemiological data regarding sexuality and PEP were collected through a standardised self-applied questionnaire. SPSS v20.0 was used for statistical analysis.

Results We enrolled 362 students, 85\% (305/359) females, mean age of $22.9( \pm 4.8)$ years, 83.1\% (301/362) self-declared non-white, 90.6\% (327/361) single and 43.4\% (154/355) had an income $\leq 2$ minimum wages. As for sexual behaviour, $12.1 \%(19 / 177)$ have sexual intercourse with people of the same sex, 22.6\% (63/279) have anal sex, 69.2\% (193/279) oral sex; $34.9 \%(96 / 275)$ irregular condom use, including $36.2 \%(81 / 224)$ of Health students $(p=0.36)$. As for the PEP, $39.3 \%(142 / 361)$ have heard about it, $73.6 \%(14 / 19)$ of the men who have sex with men had some knowledge $(\mathrm{p}<0.01$; OR 4.73, 95\% CI 1.67-13.45); 92.0\% (332/361) did not know the time limit to start the PEP, 93.1\% (335/360) did not know the duration and $68.9 \%(248 / 360)$ are unaware of the basic indication criteria; 87.0\% (315/360) did not know the sites for administration, $0.6 \%$ (2/361) affirm to have used it and $98.3 \%(348 / 354)$ state to not change the sexual behaviour after PEP, some said they would begin unprotected sexual practices, of which, $83.3 \%(5 / 6)$ were from the Health courses.

Conclusion The results evoke the challenges regarding the implantation of new strategies for HIV prevention. The limited knowledge regarding intervention and sites that offers PEP evidences barriers to access and prevention of new infections. These findings highlights the need for disseminate knowledge on PEP among young people, thus making it effective as an HIV prevention strategy.

\section{P4.108 INCREASING HIV TESTING OF EMERGENCY DEPARTMENT (ED) PATIENTS WITH INCREASED RISK FOR HIV AND THEIR PARTNERS AND PEERS THROUGH PROVISION OF HOME HIV SELF-TESTING KITS AND LINKAGE TO INTERNET-BASED RECRUITMENT FOR HIV TESTING - A PILOT RANDOMIZATION STUDY OF A NOVEL ED INTERVENTION}

Yu-Hsiang Hsieh, Anuj V Patel, Richard E Rothman, Angie S Kim, Mary Jett-Goheen, Danielle Signer, Carl A Latkin, Charlotte A Gaydos. Johns Hopkins University, Baltimore, USA

\subsection{6/sextrans-2017-053264.603}

Introduction Many emergency department (ED) patients with significant risks for HIV accept testing when offered in the ED but otherwise do not test for HIV on a regular basis. EDs could potentially serve as a portal for increasing HIV testing for this population beyond the ED. We conducted a pilot study to determine the acceptability of home HIV self-testing (HIVST) after the ED visit.

Methods In 2016, a pilot randomised study was conducted in an urban ED in Baltimore where an HIV screening program operated. Patients who accepted ED testing and were identified as having increased risk for HIV were enrolled. Consented patients were randomised to the HIVST group (received an oral fluid HIV home test kit), or control group (received a pamphlet regarding the importance of regular HIV testing). Participants in the HIVST group were encouraged to report self-testing results using an established online HIV/STI screening website, "IWantTheKit (IWTK)". Enrollees in the HIVST group also received 5 referral cards for their partners/ peers to request HIV self-testing kit from IWTK. Phone follow-up was conducted at 1 and 3 months. Increase in HIV testing proportion was estimated as a rate ratio (RR) using a chi-square test.

Results Overall, 100 patients were enrolled and randomised. At 3 month follow-up, 20 (40\%) patients in the HIVST group reported testing for HIV vs. $7(14 \%)$ in the control group [RR: 2.9 (95\% CI: $1.3,6.1)$ or $3.2(1.6,6.6)$ excluding 30 patients lost to follow-up]. $95 \%$ of patients in the HIVST group who reported testing for HIV used the provided kit. 9 of $19(47 \%)$ patients who self-tested at home reported their results to IWTK. None reported a reactive result for HIV. $54 \%$ of enrollees in the HIVST group reported that they distributed referral cards to their partners/peers; 6 used the referral card to request HIV and/or STI testing kits.

Conclusion This novel approach to providing HIVST kits for home testing to ED patients could increase overall HIV testing rates for patients who are at high risk, but not regularly tested, and for their partners and peers.

\section{P4.109 NARCOTICS ARE CHEAPER THAN FOOD IN PAKISTAN: OUR PWIDS ARE DYING FROM AIDS}

${ }^{1}$ Zeeshan Ayyaz, ${ }^{2}$ Kate Dolan. ${ }^{1}$ Amitiel Welfare Society, Bahawalpur - Pakistan; ${ }^{2}$ University of New South Wales, Program of International Research and Training, NDARC, Sydney, Australia

\subsection{6/sextrans-2017-053264.604}

Introduction It is estimated that worldwide there are nearly 12.7 million people who inject drugs. The UNODC estimates that Pakistan has 6.7 million drug users, of whom 4 million are addicts, giving us one of the highest number in the world. The UNAIDS Pakistan estimates 100000 people are living with HIV.

Method Our Society provides NSP, condoms, medical care and is linked to Government Victoria Hospital for rehabilitation PWIDs in Bahawalpur, Pakistan.

Results In 2014, we visited 6 drug hotspots and recruited 60 PWIDs. We examined their drug use, sexual risk behaviour, attitudes, HCV and HIV testing experience. Most were males $(80 \%)$ with a mean age of 30 years (R: 15-54 year), Muslim (92\%) and illiterate (55\%). All were local Bahawalpur PWIDs, many slept on the streets (83\%) and their source of income was scavenging from garbage (88\%). PWIDs thought their parents $(53 \%)$ and the community $(56 \%)$ hated them. They first used drugs at a mean age 18 years (R: 15-54 years). Most injected Morphine tablets, Pheniramine and Diazepam liquid (90\%), many shared syringes (85\%) and want to quit drugs $(66 \%)$. They had stolen to buy drugs $(76.7 \%)$ and been arrested (26.7\%). Most have had sexual intercourse (95\%), with sex workers (38\%), had a sexual preference for females (73\%), but few used condoms (18\%) or knew about safe sex $(21 \%)$, or STIs $(33 \%)$. Over half had genital itch $(58 \%)$. Disturbingly few PWIDs knew about HCV (10\%), just one person had been tested for HCV (1.7\%), yet many were interested in being tested for HCV (85\%). Most had been tested for HIV (73\%) and many were living with HIV (52\%). Conclusion Pakistani PWIDs suffer many problems, lack access to harm reduction services and are dying from inaction. We 
recommend urgent action to address this catastrophe in the making.

\section{P4.110 FOOD INSECURITY ASSOCIATED WITH ANTIRETROVIRAL THERAPY ADHERENCE AMONG HIV-INFECTED PATIENTS IN KINSHASA}

B Bepouka, H Situakibanza, F Kiazayawoko, M Mbula. University of Kinshasa, Kinshasa Democratic republic of Congo

\subsection{6/sextrans-2017-053264.605}

Introduction Food insecurity is increasingly reported as an important barrier of patient adherence to antiretroviral therapy(ART) in both resource-poor and rich settings. The objective of this study was to determine if food insecurity is associated with poor ART adherence among HIV-positive adults in a resource-limited setting.

Methods The study was a cross-sectional, conducted in Kinshasa, Democratic republic of Congo(DRC). We randomly selected patients and 9 support structures for PLHIV, from May 2015 to August 2015. Food insecurity was measured by the Household Food Insecurity Access Scale (HFIAS). Adherence was assessed by the pharmacy refill and case adherence index.Multivariate regression was used to assess whether food insecurity was associated with ART adherence.

Results Among 400 participants, 56\% were food insecure, $30 \%$ were mildly or moderately food insecure and $70 \%$ were severely food insecure. Side effects (OR 2.230, 1.327 to $3.747, \mathrm{p}=0.002$ ), and payment of consultation (OR 1.703, 1.020 to $2.843, \mathrm{p}=0,042$ ) were also associated with poor adherence.

Conclusion household food insecurity is present in more than half of the HIV-positive adults attending ART clinics in Kinshasa, and is associated with poor ART adherence.

\section{P4.111 AN APPRAISAL OF COMPREHENSIVE KNOWLEDGE OF HIV/AIDS AMONG INDIAN WOMEN: EVIDENCE FROM NATIONAL FAMILY HEALTH SURVEY FOURTH ROUND}

Deepanjali Vishwakarma. International Institute For Population Sciences, Mumbai, India

10.1136/sextrans-2017-053264.606

In order to design the suitable HIV prevention programs that help to reduce in HIV infections especially among Indian women's, it is essential to inspect the comprehensive knowledge of HIV/AIDS and prevention methods in terms of knowledge of consistence condom use among Women's. This paper explores comprehensive knowledge of HIV/AIDS among women's across the different states of India. Data were drawn from the National Family Health Survey 2015-16 (NFHS-4). The study included data of women aged 15-49 years. Results clearly reveal that Comprehensive knowledge of HIV/AIDS was significantly decline in many states like Bihar, Karnataka, Madhya Pradesh and Manipur. Compared to men, women had less comprehensive knowledge of HIV/AIDS across the states of India. There is no relation built between women education and knowledge about HIV/AIDS, results portrays that despite of having 10 or more years of schooling women's are not aware about HIV/ AIDS. Even gap between knowledge about HIV/AIDS and consistent condom use among women is significantly high varies from 23 to 49 percent across different states of India.
Interventions are needed to build knowledge of HIV/AIDS transmission and prevention methods through mass media campaigns, and information, education and communication programs so that women can get proper knowledge.

\section{P4.112 ASSOCIATIONS OF HIV TESTING WITH ANXIETY AND STRESS: IMPLICATIONS FOR FAITH BASED HIV TESTING AND TREATMENT}

Jennifer Stewart. Johns Hopkins University School of Nursing, Department of Community Public Health, Baltimore, USA

\subsection{6/sextrans-2017-053264.607}

Introduction The African American church has recently embraced a non-traditional venues perspective by incorporating HIV testing into the formal support services provided to the community. Mental health related indicators such as anxiety and stress are often associated with a lack of HIV testing. Social support from individuals and institutions within the African American community may have an important role in reducing stress and anxiety associated with HIV testing.

Methods In this cross-sectional survey based study, we surveyed and compared the responses of the congregants from two churches which offered testing and two which did not $(n=177)$. Data were analysed with descriptive statistics, Chisquare test and multivariate logistic regression

Results We found that in churches without HIV testing anxiety was significantly higher $(\mathrm{OR}=4.60, \mathrm{p}<0.001 ; 95 \% \mathrm{CI}: 2.03$, $10.41)$ as was levels of stress $(\mathrm{OR}=6.87, \mathrm{p}<0.001 ; 95 \% \mathrm{CI}$ : $2.69,17.56)$ after controlling for gender and employment status.

Conclusion These results have implications for the important role that African American churches could have in not only offering HIV testing but in reducing associated levels of stress and anxiety. They also suggest that churches willing to incorporate HIV risk reduction programs and interventions may have more profound impacts on the mental health of at risk populations.

\section{P4.113 REACH AND ACCEPTABILITY OF AN ONLINE HIV/STI TESTING SERVICE (GETCHECKEDONLINE) AMONG GAY, BISEXUAL, AND OTHER MEN WHO HAVE SEX WITH MEN LIVING IN BRITISH COLUMBIA, CANADA}

${ }^{1}$ Joshun Dulai, ${ }^{2}$ Travis Salway, ${ }^{2}$ Kimberly Thomson, ${ }^{2}$ Devon Haag, ${ }^{3}$ Nathan Lachowsky, ${ }^{4}$ Daniel Grace, ${ }^{5}$ Joshua Edward, ${ }^{2}$ Troy Grennan, ${ }^{6}$ Terry Trussler, ${ }^{2}$ Mark Gilbert. ${ }^{1}$ Columbia University, New York, USA; ${ }^{2}$ British Columbia Centre for Disease Control, Vancouver, Canada; ${ }^{3}$ University of Victoria, Victoria, Canada; ${ }^{4}$ University of Toronto, Toronto, Canada; ${ }^{5}$ Health Initiative for Men, Vancouver, Canada; ${ }^{6}$ Community-Based Research Centre for Gay Men's Health, Vancouver, Canada

\subsection{6/sextrans-2017-053264.608}

Introduction Gay, bisexual, and other men who have sex with men (MSM) in British Columbia (BC) have a high incidence of HIV/STI, with many reporting barriers to accessing testing. An online HIV/STI testing service, Get Checked Online (GCO), was launched in 2014 to reduce these barriers. In this study we examined reach and acceptability of GCO within the MSM community.

Methods We surveyed MSM living in BC over 6 months in 2016. Participants were recruited at local pride events, bars, on the street, in sexual health clinics, through social media, 